\title{
Double-layered boron. The adsorption of gas-phase atom
}

\author{
E. V. Boroznina, O. A. Kakorina, M. B. Belonenko \\ Volggrad State University, University Ave. 100, Volgograd 400062, Russia \\ boroznina.evgeniya@volsu.ru, davletova.olesya@volsu.ru
}

DOI 10.17586/2220-8054-2018-9-3-330-334

\begin{abstract}
In this paper, we have considered hexagonal double-layered boron and its adsorption properties in particular. The main adsorption characteristics have been elucidated by using the semi-empirical quantum-chemical scheme MNDO. We have investigated both external adsorption and internal infiltration of atoms $(\mathrm{H}, \mathrm{O}, \mathrm{F}, \mathrm{Cl})$ between boron monolayers.
\end{abstract}

Keywords: Boron sheet, double-layered materials, semi-empirical research, atomic adsorption.

Received: 28 September 2017

Revised: 16 October 2017

Final revision: 4 April 2018

\section{Introduction}

Recently, two-dimensional structures have become the subject of much research interests. Graphene, boron nitride, molybendum disulfide, silicene and pyrolyzed polyacrylonitriles are the most known two-dimensional structures which have been found in a large number of application variants $[1,2]$.

However, these are insufficient to cover all needs of modern technology. So, today the world of twodimensional materials has just gotten a little more crowded. The 2D universe was extended by the "borophene" [3]. This is a new allotropic form of boron which has triangular boron lattices with hexagonal arrangement of vacancies. Borophene is predicted as an exellent electrode material with a high electrochemical performance for both Li-ion and Na-ion batteries, as a component of superconducting nanodevices but several applications of borophene still await for further research and realization $[4,5]$. Boron is a unique element. It is capable of forming a wide range of its structure modifications in the condensed state [6-9]. So, in this paper we have considered the new boron structure and its adsorption properties. It is the double-layered boron sheet. We believe that the double-layered nanomaterials may greatly enrich the nanodevice applications $[10,11]$.

\section{Double-layered boron. Research of structural modification}

There are several sheet configurations divided into several distinct categories, namely hexagonal graphene-like sheet, idealized and buckled sheets, reconstructed sheets, sheets based on the icosahedral configuration, lowsymmetry sheets, and hybrid sheets. It is to be noted that our preliminary work considered only idealized and reconstructed sheet configurations [12].

The first step of our research was to model and to find the most stable configurations of hexagonal doublelayered boron (DLB). We used the semi-empirical MNDO scheme and the model of molecular cluster (MC) [13]. The matrix elements of the Hamiltonian are given by equation 1 and 2:

$$
\begin{gathered}
F_{\mu \nu}^{A A}=\delta_{\mu \nu} U_{\mu \mu}+\sum_{\mu^{\prime}, \nu^{\prime}}^{(A)} P_{\mu^{\prime} \nu^{\prime}} \cdot\left[\left\langle\mu \nu \mid \mu^{\prime} \nu^{\prime}\right\rangle-\frac{1}{2}\left\langle\mu \mu^{\prime} \mid \nu \nu^{\prime}\right\rangle\right]+\sum_{B(\neq A)}^{(E U C)}\left[\sum_{\lambda, \sigma}^{(B)} P_{\lambda \sigma} \cdot\langle\mu \nu \mid \lambda \sigma\rangle-Z_{B} \cdot\left\langle\mu \nu \mid s_{B} s_{B}\right\rangle\right], \\
F_{\mu \lambda}^{A B}=\beta_{\mu \lambda}-\frac{1}{2} \sum_{\nu}^{(A)} \sum_{\sigma}^{(B)} P_{\nu \sigma} \cdot\langle\mu \nu \mid \lambda \sigma\rangle,
\end{gathered}
$$

$U_{\mu \mu}$ are the single-center energies, which are the sum of the kinetic energy of an electron of atomic orbital $\chi_{\mu}$ of atom $A$ and the interaction potential energy between electron and nucleus of atom $A ;\langle\mu \mu \mid \nu \nu\rangle=g_{\mu \nu}$, $\langle\mu \nu \mid \mu \nu\rangle=h_{\mu \nu}$ are the single-center two-electron Coulomb integrals and exchange integrals, responsibly; $\beta_{\mu \lambda}=$ $\frac{1}{2}\left(\beta_{\mu}^{A}+\beta_{\lambda}^{B}\right) \cdot S_{\mu \lambda}$ are the two-center one-electron core-resonance integrals; $V_{\mu \nu, B}=-Z_{B} \cdot\left\langle\mu \nu \mid s_{B} s_{B}\right\rangle$ is the potential of interaction between an distributed electron over atom $A \chi_{\mu} \chi_{\nu}$ and the core of atom $B ;(\mu \nu \mid \lambda \sigma)$ are the two-center two-electron repulsion integrals.

The hexagonal boron sheet has a planar structure with a honeycomb lattice similar to that of graphene. The lengths of $\mathrm{B}-\mathrm{B}$ bonds are $1.67 \AA[14]$. The choice of $\mathrm{MC}$ as a model of the considered structures has been 
determined primarily by the fact that within of this model is possible to quite accurately investigate a local phenomenon in the structure as an adsorption, catalysis, and the formation of point defects of the structure (local centers). The extended unit cell of a hexagonal boron sheet is shown on Fig. 1. The size of the considered clusters has been optimized to exclude the boundary effect taking into account the machine time of our calculations. We have studied a rectangular molecular cluster with $x$ and $y$ dimensions of $7 a$ and $2 \sqrt{3} a$, respectively, where $a$ is the in-plane lattice parameter of borophene. The dangling bonds at the cluster boundary have been saturated by pseudoatoms of hydrogen. The initial interplanar distance between two layers of boron was chosen as that in graphite, $3.4 \AA$; afterward, we optimized the interlayer distance to create double-layered boron. The variants of sheets' positions considered relative to each other are shown on Fig. 2. We have studied several configurations of double-layered boron. The first family of configuration has been formed by displacing one of the boron sheets in a sliding motion along the $x$ axis with displacement rates $0 a, \frac{1}{2} a$ or $\frac{1}{4} a$ (Fig. 2). The second family of configurations has been formed by displacing one of the boron sheets in a sliding motion along the $y$ axis. The coefficient of displacement was $0 a, \frac{1}{2} a, \frac{1}{4} a$ or $1 a$. The lower boron sheet is shown by a dotted line. The interplanar distance of double-layered boron has been found to be $3.68 \AA$ after quantum-chemical calculations and geometrical optimization. The values of double-layered boron cohesive energy are given in the Table 1. One can see that the structure with the coefficient of displacement 1 (along the $y$ axis) is energetically more likely. But the difference of cohesive energy of another type of configurations is negligible and falls within the error of the method, hence, all of the considered variants of double-layered boron are possible and could be stable.

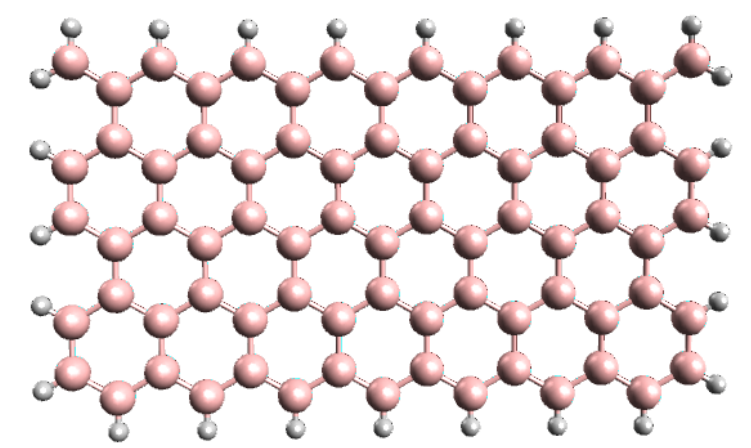

FIG. 1. The extended unit cell of molecular cluster of hexagonal boron sheet

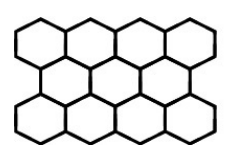

(a)

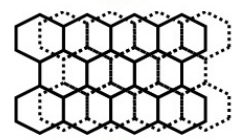

(c)

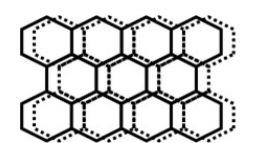

(b)

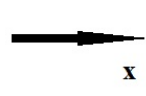

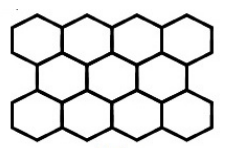

(a)

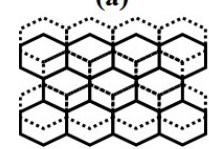

(c)

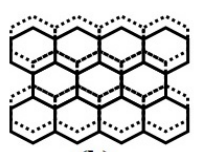

(b)

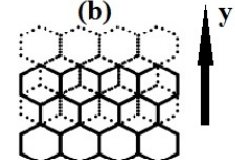

(d)

FIG. 2. Configuration of double-layered boron with $x$ axis displacement: ) without displacement; b) with the displacement rate $\frac{1}{2} a$; c) with the displacement rate $\frac{1}{4} a$. Configuration of doublelayered boron with $x$ axis displacement: ) without displacement; b) with the displacement rate $\sqrt{3} / 3 a ; \mathrm{c})$ with the displacement rate $\sqrt{3} / 6 a ; \mathrm{d})$ with the displacement rate $2 \sqrt{3} / 3 a$

\section{External adsorption of gas-phase atoms on the surface of double-layered boron}

The investigation of the binding opportunity between double-layered boron and atoms $\mathrm{H}, \mathrm{O}, \mathrm{F}, \mathrm{Cl}$ has been performed by a stepwise approach of the adsorbed atom to the surface of DLB. The potential energy of considered ad-complexes has been found on each step of our calculations. Molecular clusters of DLB with adsorbed atoms are shown on Fig. 3. The profiles of surface potential energy of the atoms interaction with the surface of DLB are shown on Fig. 4. The energy curves of these processes show a minimum which points to a chemical bond formation 
TABLE 1. Values of cohesive energy of double-layered boron $\left(E_{c}\right) . x$-displacement, $y$ - displacement

\begin{tabular}{|c|c|c|c|c|c|c|c|c|}
\hline & \multicolumn{8}{|c|}{ Rate of displacement in $a$ and $\sqrt{3} / 3 \times a$ for $x$ and $y$ directions } \\
\hline & \multicolumn{2}{|c|}{0} & \multicolumn{2}{|c|}{$1 / 2$} & \multicolumn{2}{|c|}{$1 / 4$} & \multicolumn{2}{|c|}{1} \\
\hline$E_{c}$, & $x$ & $y$ & $x$ & $y$ & $x$ & $y$ & $x$ & $y$ \\
\hline $\mathrm{eV}$ & 3.60 & 3.60 & 3.57 & 3.61 & 3.62 & 3.63 & - & 3.65 \\
\hline & & (a) & 3 & & 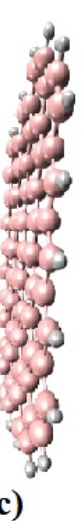 & $\begin{array}{l}3 \\
3 \\
3 \\
3 \\
\text { (d) }\end{array}$ & & \\
\hline
\end{tabular}

FIG. 3. Structures of double-layered boron with gas-phase atoms on the surface: a) hydrogen; b) oxygen; c) fluorine; d) chlorine

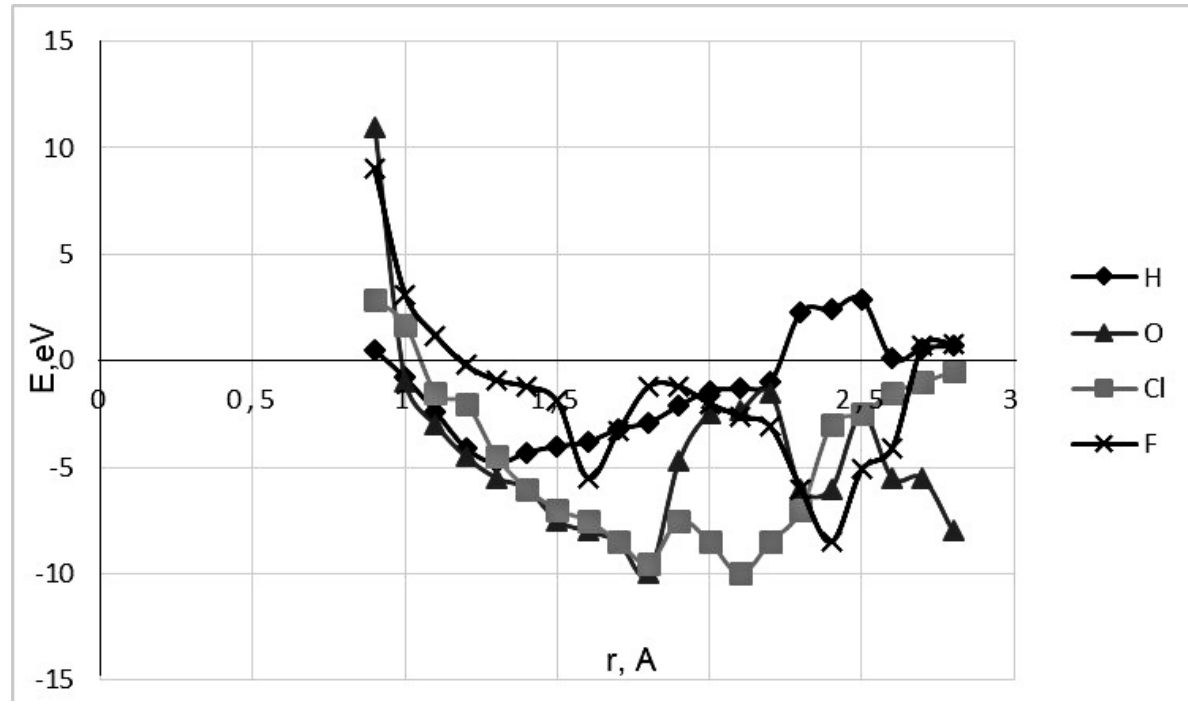

FIG. 4. Profiles of surface potential energy of the atoms interaction with the surface of doublelayered boron: a) hydrogen; b) oxygen; c) fluorine; d) chlorine

between atoms and a B atom on the surface of DLB. The adsorption energy and the distance of adsorption are given in Table 2. In the case of hydrogen atom adsorption we can see the electron density transfer from the $\mathrm{H}$ atom to the surface of the boron layer. In other words, we can conclude the fact of proton formation.

\section{Infiltration of gas-phase atoms into the interplanar space of double-layered boron}

The atoms infiltration into the interplanar space of DLB has been carried through the local vacancy of the boron sheet. The fragment of the defective boron sheet structure after total parameters optimization is shown on Fig. 5. 
TABLE 2. The basic characteristics of external DBL adsorption. $E_{a d}$ - the adsorption energy, $R_{a d}$ - the distance of adsorption

\begin{tabular}{ccccc}
\hline & \multicolumn{4}{c}{ Adsorbed atom } \\
\hline $\mathrm{H}$ & $\mathrm{O}$ & $\mathrm{F}$ & $\mathrm{Cl}$ \\
\hline$E_{a d}, \mathrm{eV}$ & -4.8 & -11.1 & -5.8 & -9.7 \\
\hline$R_{a d}, \AA$ & 1.3 & 1.8 & 1.6 & 1.8 \\
\hline
\end{tabular}

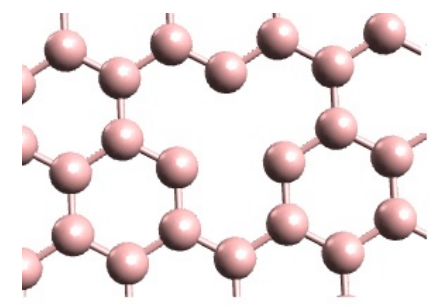

(a)

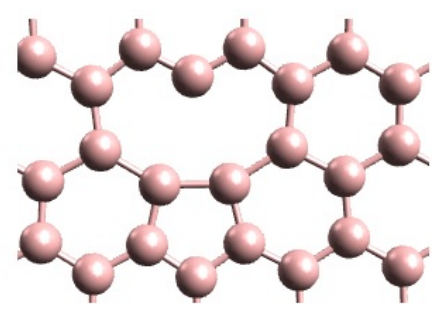

(b)

FIG. 5. Fragment of boron sheet structure with vacancy defect: a) initial structure; b) after geometry optimization

The process of infiltration was simulated by a stepwise approach (with a step of $0.1 \AA$ ) of hydrogen, oxygen, fluorine or chlorine atom along a dummy perpendicular to the surface of DLB and through the vacancy center (Fig. 6). So, the atoms have been moved from $4.5 \AA$ to $0.0 \AA$ through the DLB. The initial distance of atoms from the DLB is about $2.8 \AA$. The energy curves gives proof that the penetration of all atoms has to overcome the energy barrier. The atoms introduced have the maximum potential energy when they are lodged completely at the boron sheets vacancy (Fig. 7). Then, at inward distance of about $1 \AA$ from the defective boron layer, we can see a stable minimum of energy for all considered atoms. Hence, if the adatoms would have the possibility of overcoming the energy barrier in the classic manner, i.e. when the value of potential energy of the approaching atom is greater that the energy of potential barrier, or by tunnel effect, they will be in a stable position between two boron sheets. The heights of barriers which can be identified like the energy of infiltration activation are shown in Table 3. Hydrogen, the lightest atom, demonstrates the lowest energy activation of $3.94 \mathrm{eV}$.

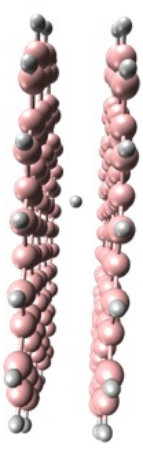

(a)

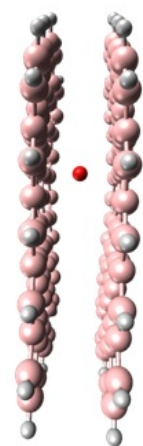

(b)

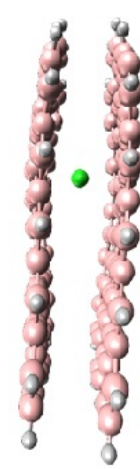

(c)

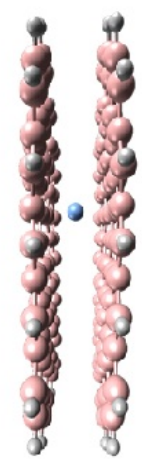

(d)

FIG. 6. Structures of double-layered boron with gas-phase atoms penetrated into the interplanar space of the double-layered boron: a) hydrogen; b) oxygen; c) fluorine; d) chlorine

\section{Conclusion}

We have found that hexagonal boron sheets can be used as a base for composites materials. It is possible to create double-layered boron and we guess that it is possible to create multi-layered materials of boron as well. 


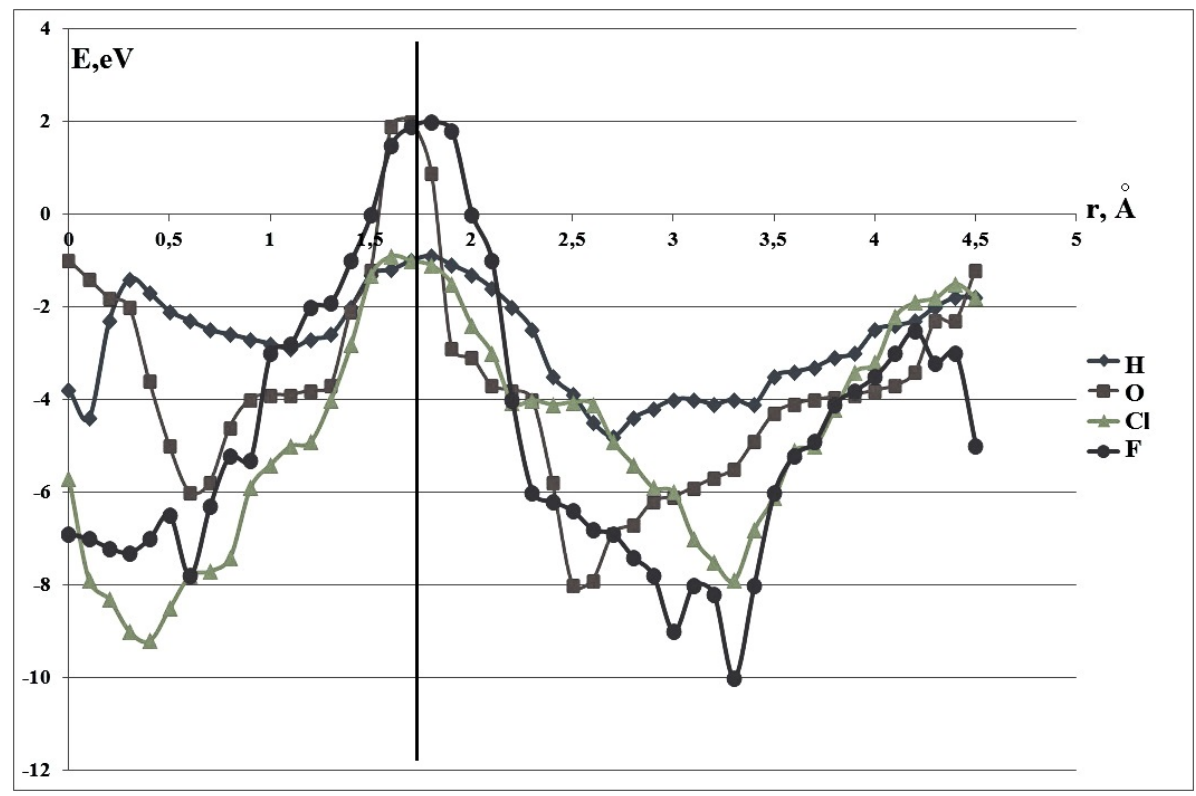

FIG. 7. Profiles of surface potential energy of the atoms infiltration into the interplanar space of the double-layered boron: a) hydrogen; b) oxygen; c) fluorine; d) chlorine. The boundary of a boron sheet with vacancy (first boron layer) has been market by vertical line

TABLE 3. Values of activation energy $E_{a c t}$ of double-layered boron for considered atoms

\begin{tabular}{ccccc}
\hline Infiltration atom & $\mathrm{H}$ & $\mathrm{O}$ & $\mathrm{F}$ & $\mathrm{Cl}$ \\
\hline$E_{\text {act }}, \mathrm{eV}$ & 3.94 & 9.92 & 6.92 & 12.44 \\
\hline
\end{tabular}

We also have found that double-layered boron has good adsorption characteristics of gas-phase atoms. One of the applications of this new nanostructure could be the hydrogen storage inside interplanar space.

\section{References}

[1] Wang K.-P., Teng H. Structural feature and double-layer capacitive performance of porous carbon powder derived from polyacrylonitrilebased carbon fiber. J. Electrochem. Soc., 2007, 154 (11), P. 993-998.

[2] Wang Y., Fugetsu B., Wang Z., Gong W. Nitrogen-doped porous carbon monoliths from polyacrylonitrile (PAN) and carbon nanotubes as electrodes for supercapacitors. Sci. Rep., 2017, 7, P. 40259.

[3] Oganov A.R., Guisinger N.P. Nanomaterials, synthesis of borophenes: Anisotropic, two-dimensional boron polymorphs. Science, 350 (6267), P. 1513-1516.

[4] Zhang X., Hu J., et al. Borophene as an extremely high capacity electrode material for Li-ion and Na-ion batteries. Nanoscale, 2016, 8 (33), P. 15340.

[5] Xiao R.C., et al. Enhanced superconductivity by strain and carrier-doping in borophene: A first principles prediction. Applied Physics Letters, 2016, 109, P. 122604

[6] Chernozatonskii L.A., Sorokin P.B., Yakcobson B.I. New boron barrelenes and tubulenes. JETP Lett., 2008, 87 (9), P. $489-493$.

[7] Penev E.S., Bhowmick S., Sadrzadeh A., Yakobson B.I. Polymorphism of two-dimensional boron. Nano Lett., 2012, 12 (5), P. $2441-2445$.

[8] Enyashin A.N., Bamburov V.G., Ivanovskii A.L. Composition, stability, and elastic moduli of higher allotropes of boron ( $\beta$-B and tII-B) according to SCC-DFTB calculations. Doklady Physical Chemistry, 2011, 438 (2), P. 118-121.

[9] Shirai K., Uemura N., Dekura H. Structure and stability of pseudo-cubic tetragonal boron. Jpn J. Appl. Phys., 2017, 56, 5 S3.

[10] Khairullin A.R., Nikolaeva M.N., Bugrov A.N. Resistance of composite films based on polystyrene and graphene oxide. Nanosystems: Phys. Chem. Math., 2016, 7 (6), P. 1055-1058.

[11] Ostroushko A.A., Russkikh O.V. Oxide material synthesis by combustion of organic-inorganic compositions. Nanosystems: Phys. Chem. Math., 2017, 8 (4), P. 476-502.

[12] Kah Chun Lau, Ravindra Pandey. Stability and electronic properties of atomistically-engineered 2D boron sheets. J. Phys. Chem. C, 2007, 111 (7), P. 2906-2912.

[13] Dewar M.J.S., Thiel W. Ground states of molecules. 38. The MNDO method. Approximations and Parameters. J. Am. Chem. Soc., 1977, 99 (15), P. 4899-4907.

[14] Boroznina E.V, Zhiganova T.A., Boroznin S.V. Reseach of vacancy defect formation on the surface of two-dimensional boron sheets. $J$. Phys.: Conf. Ser., 2015, 586, P. 012010. 